\title{
USO DE GRUPO FOCAL COMO INSTRUMENTO DE AVALIAÇÃO DE PROGRAMA EDUCACIONAL EM SAÚDE 1
}

\section{THE USE OF FOCUS GROUPS AS A TOOL TO EVALUATION OF A HEALTH EDUCACIONAL PROGRAM}

\author{
Cássia Baldini Soares* \\ Diva Reale** \\ Cristina Maria Brites***
}

\begin{abstract}
SOARES, C.B. et al. Uso de grupo focal como instrumento de avaliação de programa educacional em saúde.
\end{abstract}
Rev.Esc.Enf.USP, v.34, n.3, p. 317-22, set., 2000.

\section{RESUMO}

Este estudo objetiva identificar potencialidades e limitações do grupo focal como instrumento de avaliação através de um estudo de caso no qual o grupo focal foi utilizado para avaliar um treinamento em saúde. Entre as conclusões destacamse: é possivel comparar os resultados dos grupos realizados antes e depois do treinamento se o mesmo grupo for mantido; o grupo focal mostrou-se adequado para desvendar a natureza das deficiências do treinamento porque permitiu analisar a complexidade inerente tanto ao tema das drogas quanto aos objetivos do treinamento que busca mudanças de ordem afetivo-cultural e não apenas de ordem cognitiva.

PALAVRAS-CHAVE: Avaliação de programas. Avaliação educacional. Treinamento.

\section{ABSTRACT}

The objective of this study was to identify the possibilities and limitations of the focal group as a tool to evaluation through a case study of a health educational training process. Among the conclusions we emphasize: the maintenance of the same group turns possible to compare results of the groups accomplished before and after the training; the focal group was adequate to reveal the nature of the training deficiencies since it had permitted to analyze the ,complexity inherent to both the drug field and the training objectives that seek for cultural and affective changes and not only for cognitive changes.

KEYWORDS: Program evaluation. Educational measurement. Training.

\section{INTRODUÇÃO}

O objeto deste estudo é o grupo focal como instrumento de avaliação de programas educacionais em saúde. A avaliação constitui uma das etapas previstas no planejamento de qualquer projeto educativo que objetive mudanças seja de conhecimentos, atitudes, ou comportamentos relacionados à saúde. O campo da educação relacionada à prevenção ao uso de drogas e AIDS tem se preocupado em avaliar, sistematicamente, uma série de inovações - baseadas em diferentes abordagens teórico-metodológicas, com emprego de métodos e técnicas variados - que vêem sendo postas à prova nessa área.

O grupo focal é uma técnica que aborda os aspectos qualitativos da realidade que se quer estudar e que vem se difundindo em diferentes áreas (KRUEGER, 1988; MORGAN, 1988; MORGAN, 1993). Embora tenha origem na pesquisa de marketing, sua aplicação em outras áreas de pesquisa tem revelado potencialidades variadas:

\footnotetext{
1 Trabalhe apresentado no V Congresso Brasileiro de Saúde Coletiva e V Congresso Paulista de Saúde Pública, realizado em Águas de Lindóia - SP, de 25 a 29 de agosto de 1997, cujo conteúdo tem orientado a prática pedagógica dos autores sobre o tema.

* Enfermeira, Mestre em Saúde Pública, Doutor em Educação, Professor Doutor do Departamento de Enfermagem em Saúde Coletiva da Escola de Enfermagem da Universidade de São Paulo. E-mail: cassiaso@usp.br

** Psiquiatra, Mestre em Medicina Preventiva, Instituto de Psiquiatria da Faculdade de Medicina da Universidade de São Paulo.

***Assistente Social, Mestre em Serviço Social, Professora do Departamento de Serviço Social da Pontificia Universidade Católica - SP.
} 
desenvolvimento de programas; avaliação; pesquisa acadêmica; entre outras (FOLCH-LION; TROST, 1981; BASCH, 1987; RAMIREZ; SHEPPERD, 1988; CARLINI-COTRIM, 1996) e, ao contrário do que se imaginava nas primeiras aplicações, tem se revelado, em diferentes experiências, uma técnica adequada para pesquisar tópicos de natureza sensivel (ZELLER, 1993), inclusive na área de abuso de substâncias (CARLINI-COTRIM, 1996).

O grupo focal, conforme levantamento realizado por CARLINI-COTRIM, 1996, tem sido uma técnica utilizada tanto para ajudar na formulação de questionários, quanto em combinação com outros métodos qualitativos (entrevistas, observação participante, entre outros), ou mesmo utilizada como técnica única numa pesquisa. Embora os elementos formais que substanciam a qualidade de um grupo focal já estejam, consideravelmente, estabelecidos, recomenda-se que as novas experiências sejam acompanhadas da crítica acerca do seu uso (DEBUS, 1988).

Por isso, compusemos este trabalho em que discutimos as principais características do grupo focal, como instrumento de avaliação*, localizando suas potencialidades e limitações, a partir da análise do desenvolvimento de uma pesquisa operacional, realizada em 1997. Trata-se de um estudo, em que monitores da Fundação Estadual do Bem Estar do Menor (FEBEM) vinculada à Secretaria da Criança, Família e Bem-estar Social do Estado de São Paulo, foram treinados experimentalmente como multiplicadores em redução de danos relacionados ao uso de drogas, com a finalidade de atuar junto aos meninos e meninas de uma unidade do Município de São Paulo.

O trajeto foi sistematizado através de 5 oficinas de trabalho, cada qual com 4 horas de duração, coordenadas pelos autores deste documento** e trataram das seguintes temáticas: Oficina 1 Drogas: Mitos e Preconceitos; Oficina 2 Drogas: Conceitos básicos, Classificação e Efeitos; Oficina 3 Drogas, Adolescência e Marginalidade; Oficina 4 Drogas: Usos e Dependência; Oficina 5 Drogas: Redução de Danos. Tais oficinas foram desenvolvidas através de estratégias participativas consagradas de dinâmica de grupo (role-plays, dramatizações, entre outras) em que situações familiares do cotidiano são exploradas.
A orientação teórico-metodológica adotada foi aquela própria do campo da redução de danos que critica a ideologia e o modos operandis dominante da "guerra às drogas". Os críticos desta abordagem (CARLINI-COTRIM, 1992; COHEN, 1993; O' HARE, 1994; DUNCAN et al., 1994; BUCHER; OLIVEIRA， 1994; SOARES, 1997; WODAK, 1998) apontam que ela se fundamenta num conjunto de pressupostos moralistas, discriminatórios e estigmatizantes que contribuem para gerar e alimentar preconceitos, que conduzem a uma política e um elenco de práticas proibicionistas que favorecem o tráfico, aumentam a marginalização do usuário e utilizam métodos repressivos, amedrontadores, informações parciais $\mathrm{e}$ inverídicas que contribuem para gerar e alimentar mitos e incrementar o ceticismo.

O treinamento teve então como objetivos específicos:

1) Conceituar drogas; classificá-las segundo seus efeitos no SNC; listar os principais efeitos de acordo com as categorias.

2) Permitir a emergência dos preconceitos dos próprios participantes do treinamento.

3) Proporcionar um espaço organizado de crítica por referência aos preconceitos amplamente difundidos na sociedade em relação a: uso de drogas lícitas x ilícitas; dependência como única forma de relação indivíduo-drogas; características únicas dos usuários.

4) Introduzir o campo de idéias da redução de danos associada ao uso de drogas principalmente no que se relaciona à AIDS, um dos seus prejuízos mais nefastos ao nivel do processo saúde-doença.

5) Discutir as várias faces da adolescência , as possiveis motivações para o uso de drogas e a exclusão social em sua associação com o uso de drogas.

6) Arregimentar elementos para o estabelecimento de uma formulação crítica dos funcionários em relação ao tratamento dispensado aos usuários de droga institucionalizados na FEBEM por motivos delinqüenciais.

Tais objetivos foram formulados de modo a envolver a mobilização do treinando:1) de ordem afetiva - para compreensão da alteridade e dos processos de discriminação; 2) da ordem dos valores e atitudes - especificamente com relação

\footnotetext{
* A literatura sobre avaliação de programas educacionais vem, há algum tempo, mostrando modificações que apontam para o a composição de técnicas qualitativas e quantitativas (PARLETT; HAMILTON, 1982) para dar conta de uma interpretação mais completa da realidade empirica estudada.

** As oficinas foram coordenadas pela Dra. Diva Reale e pela Professora Cristina Maria Brites, cabendo a avaliação à Professora Cássia Baldini Soares.
} 
aos usuários de drogas; e 3) da ordem do cognitivo - especialmente no que se refere à aquisição de conhecimentos sobre drogas e sobre a adolescência. Estrategicamente, optou-se pela utilização do grupo focal como instrumento para fazer a avaliação do treinamento*. Foram planejadas duas sessões de grupo focal: uma que precedeu e outra imediatamente após o treinamento.

\section{CONSIDERAÇÕES ACERCA DOS PROCEDIMENTOS OPERACIONAIS NO USO DA TÉCNICA DE GRUPO FOCAL}

No sentido de obter algumas considerações a respeito das limitações e potencialidades do grupo focal como instrumento de avaliação através deste estudo de caso, observamos o cumprimento do trajeto operacional - tal como recomendam os estudiosos no assunto -, levando-se em conta:

1) a necessidade de se construir um roteiro de temas - guia de tópicos - para conduzir as discussões no grupo**, respeitando-se um tempo médio de uma hora e meia de trabalho.

2)a formulação de critérios de representatividade, ainda que com flexibilidade para particularizar e consolidar a composição do grupo de participantes diante de suas especificidades ${ }^{* * *}$. Recomenda-se, no entanto, que não se ultrapasse o total de 8 participantes.

3) estratégias formais de introdução no grupo, obedecendo-se às recomendações mais gerais de se proceder à identificação dos participantes através de crachá, à apresentação do moderador, do observador, dos participantes, à explicitação da finalidade do grupo e à solicitação de permissão para gravação das sessões de discussão.

4) o estabelecimento de um espaço para a sensibilização/aquecimento, etapa necessária para mobilizar o grupo.

5) o encaminhamento da discussão propriamente dita, que se inicia assim que o coordenador percebe que o grupo já está inscrito no clima da temática em pauta. Este momento deve ocupar a maior parte da sessão e só se esgota quando todos os participantes se manifestaram sobre os tópicos em debate.

6) A necessidade de se prever o "fechamento do grupo", em que o coordenador procede a uma recapitulação resumida da discussão e os participantes manifestam-se realizando as retificações que julgam convenientes e ajustadas à interpretação do que poderia ser efetivamente a opinião do grupo.

\section{CONSIDERAÇÕES ACERCA DOS PROCEDIMENTOS ANALITICOS DO MATERIAL PROVENIENTE DOS GRUPOS FOCAIS}

No sentido de complementar as considerações a respeito das limitações e potencialidades do grupo focal como instrumento de avaliação através deste estudo de caso, em particular, procedemos à análise e discussão dos conteúdos emergentes nos grupos focais realizados pré e pós-treinamento. Essa incursão foi realizada a partir das questões componentes dó guia de

*Temos utilizado essa técnica para proceder à avaliação de treinamento e de programas educacionais em outros momentos (SOARES, 1997; SOARES; SWIDER; McELMURRY, 1999).

* Guia de tópicos que orientaram as duas sessões grupais: O que entendem por drogas (psicotrópicas)? E sobre seus efeitos? Existem diferenças entre drogas lícitas e ilícitas? Existem drogas piores ou melhores? Todo mundo usa drogas do mesmo jeito? Quem são os adolescentes que usam drogas? Quais são as motivações para o uso? Como devemos tratar o usuário? O que acha que a sociedade deve fazer a respeito?***

${ }^{* * *}$ Neste estudo de caso, o grupo foi composto de 7 participantes que foram escolhidos conforme os critérios relacionados no Quadro 1: Quadro 1- Distribuição dos participantes do grupo focal segundo critérios de escolha

\begin{tabular}{|c|l|l|l|l|l|}
\hline N & Função & Unidade & Sexo & Escolaridade & Tempo de instituição \\
\hline 1 & Professor & Escola & Feminino & Sup. Inc. & -02 anos \\
\hline 1 & Auxiliar Enf. & Saúde & Feminino & $1^{\circ} \cdot$ g. C. & +05 anos \\
\hline 1 & Monitor & Semi-aberta & Feminino & $2^{\circ} \cdot$ g. C. & -02 anos \\
\hline 1 & Monitor & Fechada & Masculino & $2^{\circ}$. g. C. & +10 anos \\
\hline 1 & Monitor & Fechada & Masculino & U. g. C. & +05 anos \\
\hline 1 & Instrutor & Oficina & Masculino & U. g. Inc. & +10 anos \\
\hline 1 & Monitor & Semi-aberta & Feminino & $2^{\circ} \cdot$ g. C. & +10 anos \\
\hline
\end{tabular}


tópicos que relataremos aqui, a partir da análise dos seguintes temas: conceito de droga psicotópica; causas e motivações para o uso de drogas; relação dos adolescentes com drogas; ações da sociedade sobre o problema; necessidades do grupo de treinandos* ${ }^{*}$ as circunstâncias em que o grupo focal promoveu ou constrangeu a análise e portanto as possibilidades concretas de avaliação.

\subsection{Conceito de droga psicotrópica}

A comparação dos resultados dos dois grupos permitiu ver que o treinamento promoveu a modificação do discurso dos participantes sobre o conceito de droga psicotrópica procurando, no grupo final, defini-la com cuidado de maneira a incorporar diferentes parâmetros envolvidos. Antes do treinamento o conceito de droga psicotrópica estava confuso, ora tendendo à generalização no sentido de incluir todos os medicamentos, ora fazendo distinção a partir do conceito de dependência e assim por diante.

O encaminhamento do grupo focal permitiu ainda que os treinandos mostrassem com clareza após o treinamento que estabeleciam corretamente a distinção entre droga lícita e ilícita e que mostravam manipular, pelo menos, as diferenças básicas entre o uso ocasional e a dependência de drogas, o que não acontecia no grupo prétreinamento.

Alguns conceitos inadequados como drogas pesadas e drogas leves que tiveram presença marcante no primeiro grupo, não retornaram no grupo pós-treinamento, o que mostra que a análise comparativa do grupo pode ser feita pela dupla: presença-omissão de idéias.

\subsection{Causas e motivações para o uso de drogas}

Neste tema, a omissão de algumas palavras chaves durante o grupo final funcionou como diferenciador entre o discurso em um grupo e noutro. Por exemplo, são várias as motivações** levantadas pelo grupo de treinandos para o uso de drogas, não havendo variações relevantes entre um grupo e outro, exceto pelo fato de que a motivação:autodestruição, levantada no grupo pré-treinamento, desapareceu no grupo pós-treinamento. Falas como: quando entra no mundo das drogas penso que a pessoa está mais ou menos desesperada e tá pensando em se matar, em morrer, em se acabar, presentes de maneira veemente no primeiro grupo, não marcaram presença no segundo.

O grupo focal teve potencial também para detectar a prevalência de noções equivocadas. $\mathrm{E}$ representativo disso a fala de um dos participantes no grupo pós-treinamento, de que o uso de drogas acontece por determinação genética: acredito que venha registrado na parte genética da pessoa. Tal registro, sem dúvida, tornou-se uma questão intrigante para os organizadores merecendo algumas considerações especiais $^{* * *}$.

\subsection{Relação dos adolescentes com as drogas}

O grupo focal foi capaz de tornar perceptiveis alguns mitos e preconceitos em relação ao uso de drogas, uma vez que criou o clima para que os participantes se sentissem à vontade para expor suas opiniões. Assim, os adolescentes que eram vistos como vulneráveis a estabelecer uma relação prejudicial com as drogas devido a características de sua personalidade foram situados, no grupo realizado pós-treinamento, num contexto social indutor desse tipo de relação. Os treinandos consideraram também que na adolescência, a curiosidade é maior e que os jovens têm menos maturidade para controlar uma relação não prejudicial com a droga. A família e a formação educacional são enfatizadas na determinação da relação dos adolescentes com as drogas.

Ao relacionar o uso de drogas com os adolescentes os treinandos levantam uma reflexão que aponta a relação com a droga como um "desvio". A responsabilidade por esse "desvio" é, num primeiro momento atribuída ao adolescente e qualificada como "anormalidade": Num segundo momento, pós-treinamento, os contextos sociais são incluídos na análise e as culpabilizações individuais são relativizadas. Essa análise mostrou novamente

\footnotetext{
*Este último tema não estava previsto sendo acrescentado posteriormente por força do seu aparecimento durante a realização dos grupos focais.

** Entre as motivações levantadas nos dois grupos aparecem: busca por uma situação de prazer; fuga da realidade; curiosidade; uma impulsão para vivenciar situações novas ou de risco; desinibição; problemas interiores; momentos de depressão; necessidade de fazer o que proibido; querer ser igual ao grupo; entre outras. São bastante enfatizadas: a rejeição e a falta de estrutura familiar.

*** Para os organizadores, esse tipo de resultado levanta várias hipóteses sobre o treinamento: primeiro pode demonstrar que o enorme enraizamento dessa postura não consegue ser abalado por uma situação de treinamento muito dinâmico e que requer a rápida captação e conexão intelectual de vários conceitos. Esse resultado sugere também que pode haver necessidade de um trabalho de educação continuada para que conceitos científicos básicos sejam esclarecidos e trabalhados com mais tempo e dedicação. As colocações demonstram também a pertinência de se discutir o tema no treinamento que não tocou especificamente nessa questão apostando em elaborações mais complexas.
} 
uma potencialidade de grupo focal, pois novos discursos foram criados, mais complexos e densos, utilizando fundamentalmente os conhecimentos adquiridos durante o treinamento.

\subsection{Ações da sociedade sobre o problema}

O grupo focal revelou que, na sua maioria, os treinandos ganharam recursos de raciocinio macrosocial para encarar o problema do uso e do usuário de drogas: absorveram, por exemplo, a discussão sobre a nova lei anti-drogas que diferencia o usuário do traficante; as influências decisivas do mercado das drogas sobre o uso; os contextos de miséria e negligência social em que vivem a maior parte dos nossos jovens que criam marginalidade e violência, entre outros aspectos levantados no grupo focal ao final do treinamento.

\subsection{Sobre as necessidades do grupo de treinandos}

A discussão nos dois grupos focais fez notar que, mesmo nesse ambiente de trabalho que apresenta tamanha proximidade e riqueza de experiências com o uso de drogas pela juventude, não está havendo uma discussão monitorada sobre o assunto. No entanto, foi no grupo de finalização, pós-treinamento, que houve uma tomada de consciência por parte dos treinandos que passaram a reivindicar mais que esse tipo de iniciativa como o treinamento fosse uma constante no ambiente de trabalho. Assim, a avaliação através do grupo focal permitiu sugerir uma supervisão constante desses trabalhadores para que as experiências vivenciadas no cotidiano do trabalho pudessem passar a constituir uma base de aprendizado e não de simples divagações, normalmente distorcidas.

\section{CONSIDERAÇÕES FINAIS: POSSIBI- LIDADES E LIMITES DA TÉCNICA DO GRUPO FOCAL EM AVALIAÇÃO DE PROGRAMAS EDUCACIONAIS}

Cumprindo os passos que estruturam o trabalho em grupo focal e baseando-se na avaliação - análise de conteúdo - do caso estudado , é possível afirmar que:

1) 0 estabelecimento de critérios particulares para compor o grupo, respeitando-se o caráter da representatividade que se deve considerar ao selecionar os participantes, é condição fundamental para o andamento do grupo focal, pois se constitui um elemento facilitador de entrosamento, minimizando os constrangimentos individuais efavorecendo o respeito "à opinião do outro".
No nosso caso, em que o grupo foi propositalmente estruturado de modo a não haver diferenças de ordem hierárquica, os participantes expressaramse livremente e isentos de inibições.

2) A utilização do grupo focal, quando coincidente com a abertura do treinamento que se vai avaliar, cria um clima favorável para que os treinandos se concentrem no tema e se expressem livremente sobre a temática. Assim sendo, além de cumprir seus objetivos específicos de consolidar um primeiro momento da avaliação, a técnica simultaneamente se presta à mobilização para o treinamento. No caso aqui tomado como referência, os treinandos expressaram-se sobre mitos e preconceitos em torno das drogas e dos usuários de drogas, mesmo antes de serem foco das oficinas específicas.

3) 0 trabalho sistemático de transcrição das opiniões expressas que ela preconizacomplementado pelas observações organizadas que integram o trabalho do observador - conferem rigor e fidelidade às opiniões expressas, constituindose em ferramenta indispensável para a análise e sistematização da discussão. No nosso caso, além de ter favorecido a inclusão de temas não previstos, esta possibilidade foi potencializada pois ao proceder à análise comparativa das opiniões manifestas nas duas sessões de avaliação, observamos modificações manifestas de diferentes maneiras: por omissão de conceitos ou posturas; por arrefecimento ou relativização de posições anteriormente marcadas, substituindo termos carregados de conotações negativas por termos menos carregados; pela clara explicitação de conceitos diferentes, entre outros.

4) 0 fechamento do grupo estrategicamente planejado permite a reelaboração de alguns conceitos e posicionamentos, favorecendo a consolidação da opinião avaliativa, tocando inevitavelmente conteúdos do próprio treinamento, permitindo assim detectar pontos positivos do conteúdo a ser desenvolvido. Da mesma forma, permite perceber limites deste mesmo conteúdo, pela emergência ou manutenção de conceitos ou posturas inadequados em relação ao tema tratado. Assim sendo, a técnica guarda limitações para aquilatar aquisição de conhecimento. No nosso caso, a técnica do grupo focal permitiu analisar os problemas que se avolumam ao tratar temas que guardam complexidade tanto inerente ao tema das drogas, quanto aos próprios objetivos do treinamento que buscava mudanças de ordem afetivo-cultural e não apenas de ordem cognitiva.

5) A programação de mais de uma sessão, e estrategicamente a programação de uma sessão 
inicial e uma, ao final do treinamento, possibilita a reordenação dos temas a serem tratados e permite captar a capacidade argumentativa pela exposição seqüencial de argumentos e contra-argumentos que expressam a absorção de novos conceitos e possível modificação de antigos. Destaque-se porém que, para tanto, é preciso que o mesmo grupo seja alvo da discussão nas sessões que forem programadas, uma vez que o que interessa são as mudanças discursivas de opiniões pessoais, não se tendo a pretensão de fazer extrapolações estatísticas.

\section{Agradecimento}

À Professora Maria Josefina Leuba Salum por suas sugestões na elaboração deste trabalho.

\section{REFERÊNCIAS BIBLIOGRÁFICAS}

BASCH, C.E. Focus group interview: an underutilized research technique for improving theory and practice in health education. Health Educ. Q., v.14, n.4, p.411-8,1987.

BUCHER, R.; OLIVEIRA, S.R.M. O discurso do "combate às drogas" e suas ideologias. Rev. Saúde Públ., v.28, n.2, p.137-45, 1994.

CARLINI-COTRIM, B. A escola e as drogas: realidade brasileira e contexto internacional. São Paulo. 1992. 245p. Tese (Doutorado) - Departamento de Psicologia Social, Pontificia Universidade Católica.

CARLINI-COTRIM, B. Potencialidades da técnica qualitativa grupo focal em investigações sobre abuso de substâncias. Rev. Saúde Públ., v. 30, n. 3, p. 285-93, 1996.

COHEN, J. Achieving a reduction in drug-related harm through education. In: HEATHER, N.; WODAK, A.; NADELMANN, E.; O'HARE, P. (eds.). Psychoactive drugs and harm reduction: from faith to science. London, Whurr, 1993. p.6576.

DEBUS, M. Manual para excelencia en la investigacion mediante grupos focales. HealthCom/U.S. Agency for International Development/Academy for Educational Development, 1988.

DUNCAN, D. et al. Harm reduction: an emerging new paradigm for drug education. J. Drug Educ., v. 24, n. 2, p. 281-90, 1994.

FOLCH-LION, E.;TROST, J.F. Conducting focus group sessions, Stud. Fam. Plan., v.12, n.12, p.443-9, 1981.

KRUEGER, R. A . Focus group: a practical guide for applied research. Newbury Park, Sage, 1988.

MORGAN, D.L. Focus groups as qualitative research. Newbury Park, Sage, 1988.

MORGAN, D.L. (ed.) Successful focus group: advancing the state of the art, Newbury Park, Sage, 1993.
O'HARE, P. Redução de danos: alguns princípios e ação prática. In: MESQUITA, F.; BASTOS, F.I. (orgs.) Drogas e AIDS - estratégias de redução de danos. São Paulo, Hucitec, 1994. p. 65-78.

PARLETT, M.; HAMILTON, D. Avaliação iluminativa: uma nova abordagem no estudo de programas inovadores. In: GOLDBERG, M.A.A.; SOUZA, C. P. (org.) Avaliação de programas educacionais: vicissitudes, controvérsias e desafios São Paulo, EPU, 1982. p.38-45.

RAMIREZ, A. G.; SHEPPERD, J. The use of focus group in health research, Scand. J. Prim. Health Care, , p.81-90, 1988. Supll. 1

SOARES, C.et al. The Training of community health advocates for urban U.S. Communities: A program evaluation. In: McEIMURRY, B; TYSKA. C.; PARKER, R.S. (eds.) Prymary health care in urban communities. New York, NLN/Jones and Bartlet, 1999. Chap. 3, p.35-53.

SOARES, C.B. Adolescentes, drogas e AIDS: avaliando a prevenção e levantando necessidades. São Paulo. 284p. 1997. Tese (Doutorado) - Faculdade de Educação, Universidade de São Paulo.

WODAK, A. Redução de danos e programas de troca de seringas. In: BASTOS, F.I.; MESQUITA, F.; MARQUES, L.F. Troca de seringas: ciência, debate e saúde pública. Brasília, Ministério da Saúde, Coordenação Nacional de DST e Aids, 1988.

ZELLER, R. A. Focus group research on sensitive topics: setting the agenda without setting the agenda. In: MORGAN, D.L. Successful focus group: advancing the state of the art., Newbury Park, Sage, 1993. p. 167-83. 